\title{
Effect of Air Pollutants on Urban Roadside Trees in Shivajinagar \& Pashan Area, Pune City
}

\author{
Erum Kazi, ${ }^{1,}{ }^{*}$ Yaseen I. Shaikh, ${ }^{2}$ Suvarna Tikle, ${ }^{3}$ Gufran Beig, ${ }^{3}$ Viquar S. Shaikh, ${ }^{4}$ Satish Kulkarni ${ }^{5}$ and Vinayak P. Dhulap ${ }^{6}$
}

\begin{abstract}
Roadside urban trees have great potential to control air pollution. This study evaluates the impact of air pollutants (PM 10 \& 2.5, $\mathrm{SO}_{\mathrm{x}}, \mathrm{NO}_{\mathrm{x}}$ ) on the morphology of Polyalthia longofolia (Ashoka tree) \& Ficus racemosa (Umbar tree) in Shivajinagar and Pashan area of Pune city. APTI (Air Pollution Tolerance Index) was calculated by estimating pH, R.W.C. (Relative water content), Ascorbic acid (A.A.) \& Total chlorophyll (T.C.). The result reveals that the various oxides studied are within the permissible limits as suggested by NAAQS (National Ambient Air Quality Standard) and WHO (World Health Organisation). However, PM 10 \& PM 2.5 were found to be above the permissible limit standards prescribed by NAAQS and WHO. Ficus racemosa was found to be intermediate to air pollution, and Polyalthia longofolia was tolerant of air pollution in Shivajinagar and Pashan areas. Thus Ficus racemosa \& Polyalthia longofolia can be planted to mitigate air pollution.
\end{abstract}

Keywords: PM 10 (Particulate matter 10); PM 2.5, (Particulate matter 2.5); APTI (Air Pollution Tolerance Index); Polyalthia longofolia; Ficus racemose.

Received: 30 April 2021; Accepted: 23 July 2021.

Article type: Research article.

\section{Introduction}

The atmosphere we live in is composed of biotic and abiotic factors. Life is evolved from abiotic factors, like ammonia, carbon dioxide and water, and the same has been proved through Miller's experiment. For the perpetuation of life on the planet, the adequate composition of the abiotic factor is quite essential. The abiotic factor includes gases, moisture, light, humidity and temperature. As we know, without oxygen, life cannot be imagined on the planet; therefore, vital gases like oxygen should be maintained at an adequate level, whereas the concentration of hazardous gases should not increase beyond a critical level. Atmospheric pollution can

\footnotetext{
${ }^{l}$ Dept. of Environmental Science, Abeda Inamdar Senior College,

Pune, India.

${ }^{2}$ Dept. of Chemistry, Abeda Inamdar Sr. College, Pune

3 Indian Institute of Tropical Meteorology, Ministry of Earth

Sciences, India.

${ }^{4}$ Advanced Physics Laboratory, Department of Physics, Savitribai

Phule Pune University, Pune.

${ }^{5}$ Dept. of Environmental Science, New Arts, Commerce and

Science College, Ahmednagar, India.

${ }^{6}$ Dept. of Environmental Science, School of Earth Sciences, PAH,

Solapur University, Solapur.

*Email: environmentalisterum@gmail.com (E. Kazi)
}

create an increased level of health and life loss within a short period. Indian has witnessed the lethal effect of methyl isocyanides leakage from the union carbide company during 1984. ${ }^{[1]}$ This incidence is popularly known as the "Bhopal gas tragedy", which resulted in the death of about 8000 people, and about 500,000 were affected. Increased environmental pollution, urbanization, use of automobile motor, vehicles and industrial waste are the primary source for the increase in the concentration of gases above the threshold level. Several diseases result from pollution..$^{[2]}$ According to one survey by WHO, seven million deaths have been taken place due to pollution. ${ }^{[3]}$ Air quality control is the central aspect of environmental monitoring. This is because respiratory organ diseases are mainly ascribed to poor air quality. The significant diseases resulting from air pollution are lower respiratory infections, stroke, lung cancer and, ischemic heart diseases. Gas sensing devices are used to protect plants and personnel from inflammable and toxic gases. ${ }^{[3]}$ As gas can adversely affect humans and belongings in three main ways, as described below:

(1) Some gases are inflammable, i.e., they can catch fire at room temperature or slightly above it, e.g., methane, butane and propane.

(2) Toxic: Some gases are toxic. They can hazard humans and belongings due to their toxic nature. e.g., carbon 
monoxide, hydrogen sulfide, chlorine, and methyl isocyanide.

(3) Asphyxiant: We all need oxygen for respiration. The percentage of oxygen in the air is $20.9 \%$. When the oxygen level becomes lower than $19.5 \%$, it is deliberated oxygen deficient. When oxygen concentration drops below $16 \%$, it becomes unsuitable to humans for respiration. Some gases are having a risk of suffocation. Overexposure to Nitrogen leads to a deficiency of oxygen, and suffocation takes place.

A healthy life needs to have fresh air. Many of us may have experienced a suffocation-like situation in the crowded populations in metropolitan cities. Therefore, air quality has become a significant concern globally, especially in developing countries like India. ${ }^{[4]}$ Several anthropogenic local sources of emissions deteriorate the air quality. ${ }^{[5]}$ Pune is one of the significant developing cities that has been affected due to air pollution. ${ }^{[6]}$ There is a rise in emissions of oxides of Sulfur, Nitrogen, PM 10, and PM 2.5 in Pune City due to various developmental activities, urbanization, and increased vehicles. Air pollutants, both gaseous and particulate deteriorating air quality, produce a measurable effect on plants. ${ }^{[7]}$ It has been observed to absorb pollutants and dust particles. ${ }^{[8]}$ As surface area increases site for absorption increases; thus, the leaves with larger surface areas provide a higher site for absorption.

In the physiological process of photosynthesis, the plants synthesize complex molecules of carbohydrates from simple inorganic molecules like $\mathrm{H}_{2} \mathrm{O}$ and $\mathrm{CO}_{2}$. This process converts the physical form of the energy into a chemical form of energy. This process takes place in the presence of light and Chlorophyll. Therefore, the plants act as a sink for various gaseous and particulate pollutants. The plants store carbon dioxide as biomass and minimize the concentration of the same in the air. Simultaneously, during the physiological process of photosynthesis, oxygen gas evolves, which is very beneficial for the animal kingdom. As per the guidelines, biochemical parameters like leaf $\mathrm{pH}$, relative water content, total chlorophyll content, and APTI are the most popular index used for screening tolerance. ${ }^{[9]}$ Tree species for this proposal are used for analyzing the impact of dust deposition on plant leaves. ${ }^{[8-10]}$ APTI provides an understanding of the response of plants toward air pollution at physiological and biochemical levels, which indicate the potential of vegetation to encounter air pollution. ${ }^{[11,12]}$ This is mainly based on four significant properties of leaves, specifically the content of ascorbic acid, relative water content, total chlorophyll content, and $\mathrm{pH}$ of leaf extract. ${ }^{[13]}$

The response and sensitivity in some plants may be tolerant, while others may be sensitive to air pollution. ${ }^{[11]}$ The plant species which are more sensitive act as biological indicators of air pollution to select, rank, and screen plant species concerning their susceptibility and tolerance to air pollutants. ${ }^{[11,14]}$ Among various strategies of controlling atmospheric pollution, absorption of gaseous pollutants by plants provides one of the natural ways of cleansing the atmosphere. ${ }^{[15]}$ They act as effective indicators of air pollution. ${ }^{[16]}$

Our current study aims to study the impact of $\mathrm{SO}_{\mathrm{x}}, \mathrm{NO}_{\mathrm{x}}$, PM 10 \& PM 2.5 on roadside plants in Pashan \& Shivajinagar area. This study will help in the future to identify trees that can be planted to control air pollution in these areas. The present work aims to understand the association of air pollutants with various biochemical parameters of plants.

\section{Material and Methods}

\subsection{Study area and Selection of Plant species}

The present study has been conducted in the suburban areas of Pune, India $\left(18^{\circ} 32^{\prime} \mathrm{N}, 73^{\circ} 51^{\prime} \mathrm{E}\right.$, and altitude 559 $\mathrm{m})^{[17]}$ (Fig. 1). Pune is the seventh-largest hillocky metropolis in India and the second-largest in Maharashtra (http://www.maharashtratourism.gov.in/) with lesser air pollution. It is located on the Deccan plateau, on the leeward side of the Western Ghats, at an average elevation of 560 meters above sea level. It has a population of around 6.2 million people and is situated at the confluence of the Mula and Mutha rivers, between latitudes $18^{\circ} 25^{\prime} \mathrm{N}$ and $18^{\circ} 37^{\prime} \mathrm{N}$ and longitudes $73^{\circ} 44^{\prime} \mathrm{E}$ and $73^{\circ} 57^{\prime} \mathrm{E}$. The climate in Pune is primarily dry, with a hot semi-arid climate. Summer, monsoon, and winter are the three distinct seasons in Pune. March to May is typical summer months, with daily average temperatures varying from $30^{\circ} \mathrm{C}$ to $39{ }^{\circ} \mathrm{C}$ and relatively cool nights. The city receives an annual rainfall of $722 \mathrm{~mm}$, mainly between June and September. The winds are generally light to moderate, increasing the force during the monsoon season during November and December. The winds are generally calm or flow mainly from the east or southeast. The westerly's and south westerly's are more common during the late summer and monsoon seasons.

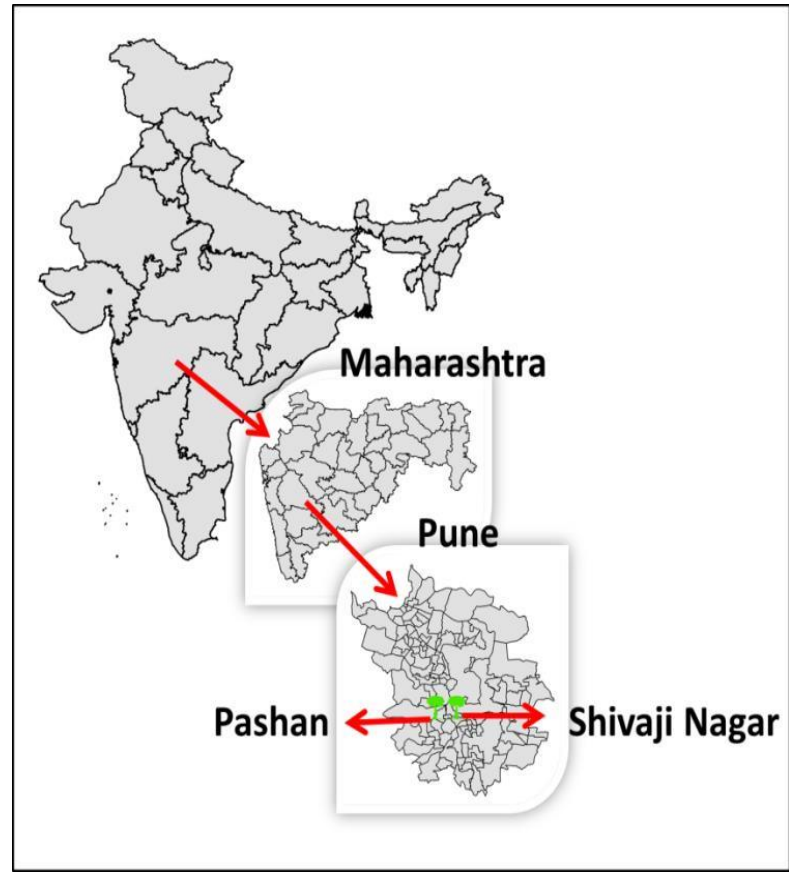

Fig. 1 Location map. 


\begin{tabular}{llllllllllllllllllllllll}
$E$ & $S$ & & $F$ & $o$ & $o$ & $d$ & & $\&$ & & $A$ & $g$ & $r$ & $o$ & $f$ & $o$ & $r$ & $e$ & $s$ & $t$ & $r$ & $y$ \\
\hline $\mathrm{R}$ & $\mathrm{e}$ & $\mathrm{s}$ & $\mathrm{e}$ & & $\mathrm{a}$ & & $\mathrm{r}$ & $\mathrm{C}$ & $\mathrm{h}$ & & & $\mathrm{a}$ & & $\mathrm{r}$ & & $\mathrm{t}$ & $\mathrm{i}$ & $\mathrm{c}$ & & & $\mathrm{e}$
\end{tabular}

Pune city experiences moderate air quality. Winter and summer seasons show an increase in air pollution levels, and monsoon season shows a decline in air pollutant levels. Pune city ranked $299^{\text {th }}$ dirtiest city in the world in 2019. Residents have experienced itchy skin, watery eyes and irritated respiratory tract problems. Doctors in the city have reported a $30-40 \%$ increase in Upper respiratory tract infection (URTI). Significant causes of air pollution in the city are a rapid expansion of the city area, construction activities, and increased motor vehicles which expels carbon dioxide every day and causes the Greenhouse effect.

Shivajinagar is situated on the west bank of the Mutha River, with the older parts of Pune on the east side of the river. The area is surrounded on its western side by the Vetal and Hanuman Hills. Shivajinagar is well connected to other parts of the city by local PMPML buses. Shivajinagar has a Shivajinagar railway station. Pashan is a suburb of Pune, India. It is located off the Mumbai-Bangalore national highway, by-passing Pune city. Pashan road serves as the main approach road for the Mumbai-Pune expressway.

To study the distribution of plants growing alongside the road in detail, a survey has been carried out to screen the most tolerant plant species to pollution. To maintain uniformity, plants of the same age and growing at iso-ecological conditions were selected for the study. Two common plant species growing at polluted location Shivajinagar and greener location Pashan have been selected viz. Ficus racemosa (Umbar) and Polyalthia longifolia (Ashoka) for comparative study.

Fully mature leaves of almost the same age and size (i.e., 4th from the tip) were collected in morning hours from the selected trees during pre-monsoon and post-monsoon season in 2018. The samples from each site were obtained with the utmost caution from plants growing in iso-ecological conditions of light, water, and soil. A composite sample of each plant species was collected and sent to the laboratory for study.

\subsection{Monitoring of Air Pollutants}

Air quality data was obtained from System of Air Quality and Weather Forecasting and Research (SAFAR). ${ }^{[18-20]}$

\subsection{Analysis of leaves for biochemical parameters:}

Leaf samples were analyzed for four biochemical parameters (Total Chlorophyll, Relative water content, $\mathrm{pH}$, and Ascorbic acid using standard methods (Table 1). By combining these parameters, the 'Air pollution Tolerance Index' (APTI) was computed by the method ${ }^{[21,22]}$ using the following Eq. 1.

$$
\mathrm{APTI}=[\mathrm{A}(\mathrm{T}+\mathrm{P})]+\mathrm{R} / 10
$$

where, APTI: Air pollution tolerance Index, $\mathrm{A}=$ ascorbic acid content of leaf in $\mathrm{mg} / \mathrm{g}$ dry weight $\mathrm{T}=$ total chlorophyll content of leaf in $\mathrm{mg} / \mathrm{g}$ dry weight $\mathrm{P}=$ Leaf extract $\mathrm{pH}, \mathrm{R}=$ Relative water content (R.W.C.), $10=$ Total sum is divided by 10 to obtain APTI values. Classification of plants into different categories of tolerance levels has been done as per
Singh S.K. and Rao (1991). ${ }^{[22]}$ APTI between 30-100 was termed as Tolerant, 29-17 were termed Intermediate, and plants with APTI between 16-1 were termed as Sensitive to Air Pollution.

Table 1. Showing Biochemical Parameters used for Plant analysis \& Methods used.

\begin{tabular}{ccc}
\hline Sr. No. & Parameters & Biochemical Method \\
\hline 1 & Total Chlorophyll & Arnon $(1949)^{[30]}$ \\
2 & Relative water content & A. Singh $(1977)^{[31]}$ \\
3 & pH & A. Singh $(1977)^{[31]}$ \\
4 & Ascorbic acid & B. Aberg $(1958)^{[32]}$ \\
\hline
\end{tabular}

Table 2. WHO standard values.

\begin{tabular}{ccc}
\hline Pollutant & $\begin{array}{c}\text { Time Weighted } \\
\text { Average }\end{array}$ & $\begin{array}{c}\text { Concentration in } \\
\text { Ambient Air }\end{array}$ \\
\hline $\begin{array}{c}\text { Particulate matter (size } \\
\text { less than } 2.5 \mu \mathrm{m} \text { ) or }\end{array}$ & $\begin{array}{c}\text { Annual mean } \\
\text { 24-hour mean }\end{array}$ & $10 \mu \mathrm{g} / \mathrm{m}^{3}$ \\
PM2.5 $\mu \mathrm{g} / \mathrm{m}^{3}$ & & \\
Particulate matter (size & Annual mean & $20 \mu \mathrm{g} / \mathrm{m}^{3}$ \\
less than $10 \mu \mathrm{m}$ ) or & 24-hour mean & $50 \mu \mathrm{g} / \mathrm{m}^{3}$ \\
PM10 $\mu \mathrm{g} / \mathrm{m}^{3}$ & & \\
Nitrogen Dioxide & Annual mean & $40 \mu \mathrm{g} / \mathrm{m}^{3}$ \\
$\left(\mathrm{NO}_{2}\right), \mu \mathrm{g} / \mathrm{m}^{3}$ & 1-hour mean & $200 \mu \mathrm{g} / \mathrm{m}^{3}$ \\
Sulphur Dioxide $\left(\mathrm{SO}_{2}\right)$, & 24-hour mean & $20 \mu \mathrm{g} / \mathrm{m}^{3}$ \\
$\mu \mathrm{g} / \mathrm{m}^{3}$ & 10-minute mean & $500 \mu \mathrm{g} / \mathrm{m}^{3}$ \\
\hline
\end{tabular}

\section{Results and Discussion}

Tables 2, 3 \& 4 show the Ambient Air Quality Standards prescribed by WHO \& NAAQS. Table 5 explains that concentration of Particulate matter (PM 10) was found to be above the prescribed limit of WHO \&NAAQS in Pashan and Shivajinagar area. Rainy season showed low concentration of PM 10 concentration. Winter season showed increase in concentration of PM 10 in both Pashan \& Shivajinagar area respectively. Summer season showed slight increase in PM 10 concentrations than prescribed limit of WHO \& NAAQS in both the areas.

Table 6 explains that concentration of Particulate Matter (PM 2.5) was found to be below the permissible limit in Pashan \& Shivajinagar area in rainy season as prescribed by WHO \& NAAQS. Winter season \& summer season showed increase in concentration of PM 2.5 as per the prescribed standards of NAAQS \& low as compared to WHO standards in both the areas.

Table 7 explains that concentration of $\mathrm{NO}_{2}$ was found to be below the standards limit in Shivajinagar \& Pashan area as prescribed by WHO \& NAAQS standards. There was increase in $\mathrm{NO}_{2}$ levels from winter to summer season in both the areas, rainy season showed decline in concentration.

Table 8 explains that concentration of $\mathrm{SO}_{2}$ was found to be above the permissible limit set by WHO \& below as per NAAQS in Pashan \& Shivajinagar area. Concetration was found to be low in rainy season as compared to Winter and Summer season. 
The comparison of $\mathrm{pH}$ and T.C. shown in Fig. 2(a) observed at polluted and greener sites for the study period during $2018 \mathrm{pH}$ of the leaf extract of Ficus racemosa varied from 4.2 at Pashan to 4.6 at Shivaji Nagar (Ficus racemosa) and of Polyalthia longifolia (Ashoka tree) varied from 4.2 to 4.8. The result shows that $\mathrm{pH}$ is acidic in both trees. The highest reduction in $\mathrm{pH}$ value in polluted areas compared to cleaner areas has been observed. A similar result was obtained, suggesting that $\mathrm{SO}_{2}$ and $\mathrm{NO}_{\mathrm{x}}$ in the ambient air causes a change in $\mathrm{pH}$ of the leaf sap towards the acidic range. Air pollution showed a profoundly decreased T.C. (Total Chlorophyll Content) in both trees in polluted areas. At cleaner sites, total chlorophyll content was observed at the same amount of $0.0086 \mathrm{mg}$ g-1. Loss in chlorophyll content in plants may be due to suspended particulate matter and other pollutants in the air, and the same is reported by Tripathi et al. ${ }^{[15]}$ It can be observed that lower chlorophyll content in both plants indicates a higher sensitivity nature of the plant species. ${ }^{[23]}$ It was discovered that at Pashan Total Chlorophyll content of Polyalthia longifolia $0.00831 \mathrm{mg} \mathrm{g}^{-1}$ and Ficus racemosa was found to be $0.00809 \mathrm{mg} \mathrm{g}^{-1}$ in the Shivajinagar area. The Pashan area showed Total Chlorophyll of Polyalthia longifolia $0.00863 \mathrm{mg}^{-1}$, and Ficus racemosa also showed $0.00861 \mathrm{mg}^{-1}$.

The ascorbic acid and relative water content vary from plant to plant and location to location. The variation in ascorbic acid content and relative water content is shown in Fig. 2(b). The experimentation reveals very high ascorbic acid (A.A.) content $66.4 \mathrm{mg} / \mathrm{gm}$ and $99.6 \mathrm{mg} / \mathrm{gm}$ for Polyalthia longifolia (Ashoka tree) at Shivajinagar and Pashan, respectively. The A.A. content at Pashan of Polyalthia longifolia was found to be $99.6 \mathrm{mg} / \mathrm{gm}$ \& Ficus racemosa showed $49.8 \mathrm{mg} / \mathrm{gm}$. The A.A. concentration at
Shivajinagar of Polyalthia longifolia was found to be 66.4 $\mathrm{mg} / \mathrm{gm}$, and Ficus racemosa was found to be $49.8 \mathrm{mg} / \mathrm{gm}$. The concentration of ascorbic acid is an indicator of tolerance of the plant towards air pollution. A higher concentration of ascorbic acid in plants indicates exposure to the high concentration of $\mathrm{SO}_{2}$ and higher tolerance of the plant. ${ }^{[24]}$ The Ficus racemosa (Umbar tree) at Shivajinagar showed comparatively low Ascorbic acid content 49.8 $\mathrm{mg} / \mathrm{gm}$. Low ascorbic levels indicate sensitivity towards air

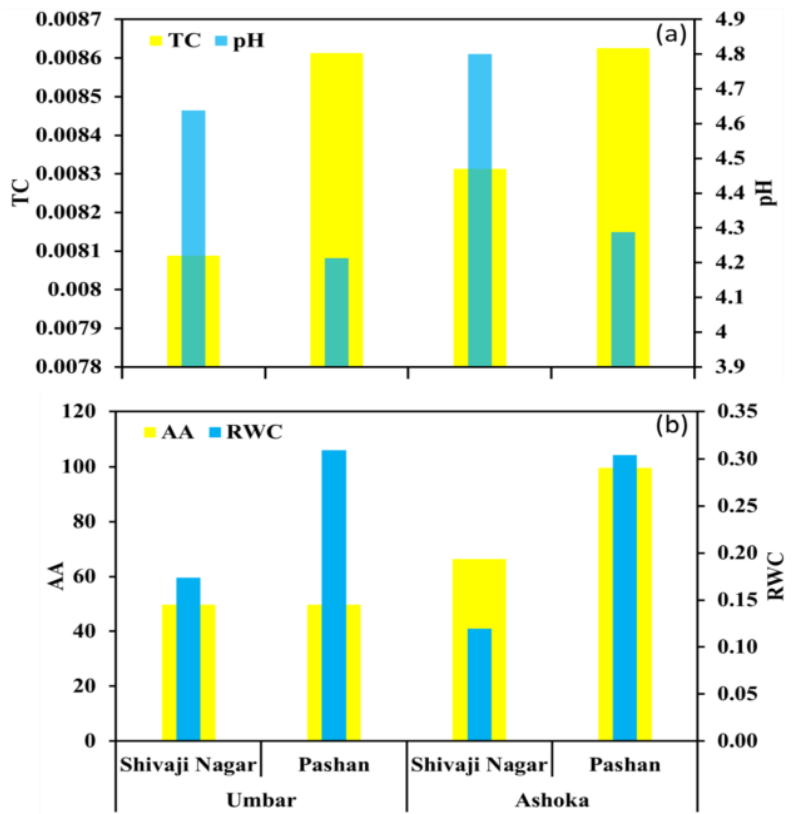

Fig. 2 (a) Impact of air pollution on Total Chlorophyll (T.C.) and $\mathrm{pH}$ (a) response of in polluted area and cleaner area, (b) Impact of air pollution on Ascorbic acid (A.A.) and Relative water content (R.W.C.) response of in polluted area and cleaner area.

Table 3. National Ambient Air Quality Standards-(NAAQS).

\begin{tabular}{|c|c|c|c|}
\hline \multirow[b]{2}{*}{ Pollutant } & \multirow[b]{2}{*}{ Time Weighted Average } & \multicolumn{2}{|l|}{ Concentration in Ambient Air } \\
\hline & & $\begin{array}{l}\text { Industrial, Residential, Rural } \\
\text { and Other Areas }\end{array}$ & $\begin{array}{ll}\begin{array}{l}\text { Ecologically } \\
\text { (notified Sensitive Area }\end{array} \\
\text { Government) }\end{array}$ \\
\hline \multirow{2}{*}{$\begin{array}{l}\text { Sulphur Dioxide }\left(\mathrm{SO}_{2}\right) \\
\mu \mathrm{g} / \mathrm{m}^{3}\end{array}$} & Annual* & $50 \mu \mathrm{g} / \mathrm{m}^{3}$ & $20 \mu \mathrm{g} / \mathrm{m}^{3}$ \\
\hline & 24 hours** & $80 \mu \mathrm{g} / \mathrm{m}^{3}$ & $80 \mu \mathrm{g} / \mathrm{m}^{3}$ \\
\hline \multirow{2}{*}{$\begin{array}{l}\text { Nitrogen Dioxide }\left(\mathrm{NO}_{2}\right), \\
\mu \mathrm{g} / \mathrm{m} 3\end{array}$} & Annual* & $40 \mu \mathrm{g} / \mathrm{m}^{3}$ & $30 \mu \mathrm{g} / \mathrm{m}^{3}$ \\
\hline & 24 hours** & $80 \mu \mathrm{g} / \mathrm{m}^{3}$ & $80 \mu \mathrm{g} / \mathrm{m}^{3}$ \\
\hline \multirow{2}{*}{$\begin{array}{l}\text { Particulate matter (size less } \\
\text { than } 10 \mu \mathrm{m} \text { ) or PM } 10 \mu \mathrm{g} / \mathrm{m}^{3}\end{array}$} & Annual* & $60 \mu \mathrm{g} / \mathrm{m}^{3}$ & $60 \mu \mathrm{g} / \mathrm{m}^{3}$ \\
\hline & 24 hours** & $100 \mu \mathrm{g} / \mathrm{m}^{3}$ & $100 \mu \mathrm{g} / \mathrm{m}^{3}$ \\
\hline \multirow{2}{*}{$\begin{array}{l}\text { Particulate matter (size less } \\
\text { than } 2.5 \mu \mathrm{m} \text { ) or PM } 2.5 \mu \mathrm{g} / \mathrm{m}^{3}\end{array}$} & Annual* & $40 \mu \mathrm{g} / \mathrm{m}^{3}$ & $40 \mu \mathrm{g} / \mathrm{m}^{3}$ \\
\hline & 24 hours** & $60 \mu \mathrm{g} / \mathrm{m}^{3}$ & $60 \mu \mathrm{g} / \mathrm{m}^{3}$ \\
\hline
\end{tabular}

Annual arithmetic mean of minimum 104 measurements in a year at a particular site taken twice a week 24 hourly at uniform intervals.

** 24 hourly or 8 hourly or 1 hourly monitored values, as applicable, shall be complied with $98 \%$ of the time, they may exceed the limits but not on two consecutive days of monitoring.

Source: National Ambient Air Quality Standards, Central Pollution Control Board Notification in the Gazette of India, Extraordinary, New Delhi, 18th November, 2009. 


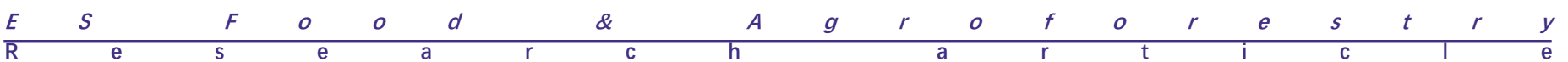

pollution, and high levels of ascorbic acid reveal tolerance towards air pollution and confer protection in plants against adverse effects of oxidative stress. ${ }^{[25]}$ It has been observed that R.W.C. of Ficus racemosa at Shivajinagar is 0.1739 $\mathrm{mg} / \mathrm{gm}$, and at Pashan, it was found to be $0.30929 \mathrm{mg} / \mathrm{gm}$. R.W.C. of Polyalthia longifolia was to be $0.11928 \mathrm{mg} / \mathrm{gm}$ at Shivajinagar and $0.30414 \mathrm{mg} / \mathrm{gm}$ at Pashan area. It shows that R.W.C. in the Shivajinagar area (Polluted area) is less than R.W.C. in Pashan (lesser polluted) area. The reduced relative water content indicates disturbed physiological status in the plants due to pollution. ${ }^{[26]}$

Table 4. Comparison between WHO standards \& NAAQS Standards.

\begin{tabular}{ccc}
\hline Pollutant & WHO standards & NAAQS Standards \\
\hline PM10 $\mu \mathrm{g} / \mathrm{m}^{3}$ & $50 \mu \mathrm{g} / \mathrm{m}^{3}$ & $80 \mu \mathrm{g} / \mathrm{m}^{3}$ \\
PM2.5 $\mu \mathrm{g} / \mathrm{m}^{3}$ & $25 \mu \mathrm{g} / \mathrm{m}^{3}$ & $80 \mu \mathrm{g} / \mathrm{m}^{3}$ \\
$\mathrm{NO}_{2}$ & $40 \mu \mathrm{g} / \mathrm{m}^{3}$ & $100 \mu \mathrm{g} / \mathrm{m}^{3}$ \\
$\mathrm{SO}_{2}$ & $20 \mu \mathrm{g} / \mathrm{m}^{3}$ & $60 \mu \mathrm{g} / \mathrm{m}^{3}$ \\
\hline
\end{tabular}

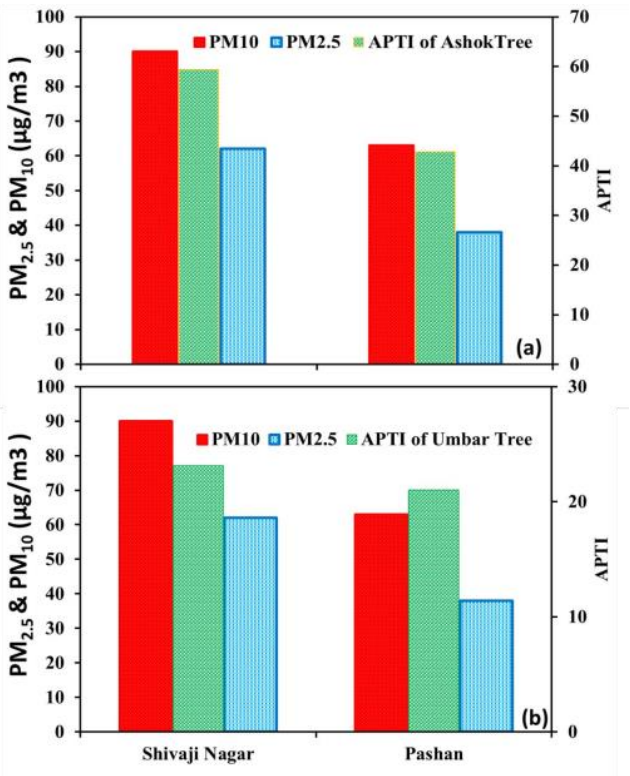

Fig. 3 APTI values and average concentration of $\mathrm{PM}_{10}$ and $\mathrm{PM}_{2.5}$ in (a) in Shivajinagar and (b) Pashan.

Table 5. PM 10 was found to be above permissible limits set by WHO in 2017 \& 2018. Higher in winter month in 2017 as per NAQS.

\begin{tabular}{llclll}
\hline \multicolumn{7}{c}{ PM 10 } & \multicolumn{3}{l}{} \\
\hline Year & Month & Shivajinagar & Pashan & WHO & NAAQS \\
\hline 2017 & May & $64 \mu \mathrm{g} / \mathrm{m}^{3}$ & $50 \mu \mathrm{g} / \mathrm{m}^{3}$ & $50 \mu \mathrm{g} / \mathrm{m}^{3}$ & $80 \mu \mathrm{g} / \mathrm{m}^{3}$ \\
2017 & June & $46 \mu \mathrm{g} / \mathrm{m}^{3}$ & $33 \mu \mathrm{g} / \mathrm{m}^{3}$ & $50 \mu \mathrm{g} / \mathrm{m}^{3}$ & $80 \mu \mathrm{g} / \mathrm{m}^{3}$ \\
2017 & July & $44 \mu \mathrm{g} / \mathrm{m}^{3}$ & $42 \mu \mathrm{g} / \mathrm{m}^{3}$ & $50 \mu \mathrm{g} / \mathrm{m}^{3}$ & $80 \mu \mathrm{g} / \mathrm{m}^{3}$ \\
2017 & August & $49 \mu \mathrm{g} / \mathrm{m}^{3}$ & $36 \mu \mathrm{g} / \mathrm{m}^{3}$ & $50 \mu \mathrm{g} / \mathrm{m}^{3}$ & $80 \mu \mathrm{g} / \mathrm{m}^{3}$ \\
2017 & September & $46 \mu \mathrm{g} / \mathrm{m}^{3}$ & $32 \mu \mathrm{g} / \mathrm{m}^{3}$ & $50 \mu \mathrm{g} / \mathrm{m}^{3}$ & $80 \mu \mathrm{g} / \mathrm{m}^{3}$ \\
2017 & October & $64 \mu \mathrm{g} / \mathrm{m}^{3}$ & $53 \mu \mathrm{g} / \mathrm{m}^{3}$ & $50 \mu \mathrm{g} / \mathrm{m}^{3}$ & $80 \mu \mathrm{g} / \mathrm{m}^{3}$ \\
2017 & November & $118 \mu \mathrm{g} / \mathrm{m}^{3}$ & $66 \mu \mathrm{g} / \mathrm{m}^{3}$ & $50 \mu \mathrm{g} / \mathrm{m}^{3}$ & $80 \mu \mathrm{g} / \mathrm{m}^{3}$ \\
2017 & December & $126 \mu \mathrm{g} / \mathrm{m}^{3}$ & $94 \mu \mathrm{g} / \mathrm{m}^{3}$ & $50 \mu \mathrm{g} / \mathrm{m}^{3}$ & $80 \mu \mathrm{g} / \mathrm{m}^{3}$ \\
2018 & January & $119 \mu \mathrm{g} / \mathrm{m}^{3}$ & $91 \mu \mathrm{g} / \mathrm{m}^{3}$ & $50 \mu \mathrm{g} / \mathrm{m}^{3}$ & $80 \mu \mathrm{g} / \mathrm{m}^{3}$ \\
2018 & February & $114 \mu \mathrm{g} / \mathrm{m}^{3}$ & $83 \mu \mathrm{g} / \mathrm{m}^{3}$ & $50 \mu \mathrm{g} / \mathrm{m}^{3}$ & $80 \mu \mathrm{g} / \mathrm{m}^{3}$ \\
2018 & March & $111 \mu \mathrm{g} / \mathrm{m}^{3}$ & $80 \mu \mathrm{g} / \mathrm{m}^{3}$ & $50 \mu \mathrm{g} / \mathrm{m}^{3}$ & $80 \mu \mathrm{g} / \mathrm{m}^{3}$ \\
2018 & April & $94 \mu \mathrm{g} / \mathrm{m}^{3}$ & $77 \mu \mathrm{g} / \mathrm{m}^{3}$ & $50 \mu \mathrm{g} / \mathrm{m}^{3}$ & $80 \mu \mathrm{g} / \mathrm{m}^{3}$ \\
\hline
\end{tabular}

Table 6. PM 2.5 Found to be above permissible limits set by WHO in 2017 \& 2018 . Below as per NAAQS.

\begin{tabular}{cccccc}
\hline Year & Month & Shivajinagar & Pashan & WHO & NAQS \\
\hline 2017 & May & $22 \mu \mathrm{g} / \mathrm{m}^{3}$ & $13 \mu \mathrm{g} / \mathrm{m}^{3}$ & $25 \mu \mathrm{g} / \mathrm{m}^{3}$ & $80 \mu \mathrm{g} / \mathrm{m}^{3}$ \\
2017 & June & $15 \mu \mathrm{g} / \mathrm{m}^{3}$ & $16 \mu \mathrm{g} / \mathrm{m}^{3}$ & $25 \mu \mathrm{g} / \mathrm{m}^{3}$ & $80 \mu \mathrm{g} / \mathrm{m}^{3}$ \\
2017 & July & $10 \mu \mathrm{g} / \mathrm{m}^{3}$ & $6 \mu \mathrm{g} / \mathrm{m}^{3}$ & $25 \mu \mathrm{g} / \mathrm{m}^{3}$ & $80 \mu \mathrm{g} / \mathrm{m}^{3}$ \\
2017 & August & $6 \mu \mathrm{g} / \mathrm{m}^{3}$ & $4 \mu \mathrm{g} / \mathrm{m}^{3}$ & $25 \mu \mathrm{g} / \mathrm{m}^{3}$ & $80 \mu \mathrm{g} / \mathrm{m}^{3}$ \\
2017 & September & $7 \mu \mathrm{g} / \mathrm{m}^{3}$ & $4 \mu \mathrm{g} / \mathrm{m}^{3}$ & $25 \mu \mathrm{g} / \mathrm{m}^{3}$ & $80 \mu \mathrm{g} / \mathrm{m}^{3}$ \\
2017 & October & $20 \mu \mathrm{g} / \mathrm{m}^{3}$ & $6 \mu \mathrm{g} / \mathrm{m}^{3}$ & $25 \mu \mathrm{g} / \mathrm{m}^{3}$ & $80 \mu \mathrm{g} / \mathrm{m}^{3}$ \\
2017 & November & $33 \mu \mathrm{g} / \mathrm{m}^{3}$ & $12 \mu \mathrm{g} / \mathrm{m}^{3}$ & $25 \mu \mathrm{g} / \mathrm{m}^{3}$ & $80 \mu \mathrm{g} / \mathrm{m}^{3}$ \\
2017 & December & $45 \mu \mathrm{g} / \mathrm{m}^{3}$ & $13 \mu \mathrm{g} / \mathrm{m}^{3}$ & $25 \mu \mathrm{g} / \mathrm{m}^{3}$ & $80 \mu \mathrm{g} / \mathrm{m}^{3}$ \\
2018 & January & $70 \mu \mathrm{g} / \mathrm{m}^{3}$ & $65 \mu \mathrm{g} / \mathrm{m}^{3}$ & $25 \mu \mathrm{g} / \mathrm{m}^{3}$ & $80 \mu \mathrm{g} / \mathrm{m}^{3}$ \\
2018 & February & $74 \mu \mathrm{g} / \mathrm{m}^{3}$ & $63 \mu \mathrm{g} / \mathrm{m}^{3}$ & $25 \mu \mathrm{g} / \mathrm{m}^{3}$ & $80 \mu \mathrm{g} / \mathrm{m}^{3}$ \\
2018 & March & $79 \mu \mathrm{g} / \mathrm{m}^{3}$ & $58 \mu \mathrm{g} / \mathrm{m}^{3}$ & $25 \mu \mathrm{g} / \mathrm{m}^{3}$ & $80 \mu \mathrm{g} / \mathrm{m}^{3}$ \\
2018 & April & $67 \mu \mathrm{g} / \mathrm{m}^{3}$ & $53 \mu \mathrm{g} / \mathrm{m}^{3}$ & $25 \mu \mathrm{g} / \mathrm{m}^{3}$ & $80 \mu \mathrm{g} / \mathrm{m}^{3}$ \\
\hline
\end{tabular}


Table 7. $\mathrm{NO}_{2}$ Found to be above permissible limits set by WHO in 2017 \& 2018. Below as per NAAQS.

\begin{tabular}{cccccc}
\hline Year & Month & Shivajinagar & Pashan & WHO & NAAQS \\
\hline 2017 & May & $22 \mu \mathrm{g} / \mathrm{m}^{3}$ & $13 \mu \mathrm{g} / \mathrm{m}^{3}$ & $40 \mu \mathrm{g} / \mathrm{m}^{3}$ & $100 \mu \mathrm{g} / \mathrm{m}^{3}$ \\
2017 & June & $15 \mu \mathrm{g} / \mathrm{m}^{3}$ & $16 \mu \mathrm{g} / \mathrm{m}^{3}$ & $40 \mu \mathrm{g} / \mathrm{m}^{3}$ & $100 \mu \mathrm{g} / \mathrm{m}^{3}$ \\
2017 & July & $19 \mu \mathrm{g} / \mathrm{m}^{3}$ & $21 \mu \mathrm{g} / \mathrm{m}^{3}$ & $40 \mu \mathrm{g} / \mathrm{m}^{3}$ & $100 \mu \mathrm{g} / \mathrm{m}^{3}$ \\
2017 & August & $12 \mu \mathrm{g} / \mathrm{m}^{3}$ & $16 \mu \mathrm{g} / \mathrm{m}^{3}$ & $40 \mu \mathrm{g} / \mathrm{m}^{3}$ & $100 \mu \mathrm{g} / \mathrm{m}^{3}$ \\
2017 & September & $14 \mu \mathrm{g} / \mathrm{m}^{3}$ & $18 \mu \mathrm{g} / \mathrm{m}^{3}$ & $40 \mu \mathrm{g} / \mathrm{m}^{3}$ & $100 \mu \mathrm{g} / \mathrm{m}^{3}$ \\
2017 & October & $40 \mu \mathrm{g} / \mathrm{m}^{3}$ & $14 \mu \mathrm{g} / \mathrm{m}^{3}$ & $40 \mu \mathrm{g} / \mathrm{m}^{3}$ & $100 \mu \mathrm{g} / \mathrm{m}^{3}$ \\
2017 & November & $66 \mu \mathrm{g} / \mathrm{m}^{3}$ & $24 \mu \mathrm{g} / \mathrm{m}^{3}$ & $40 \mu \mathrm{g} / \mathrm{m}^{3}$ & $100 \mu \mathrm{g} / \mathrm{m}^{3}$ \\
2017 & December & $80 \mu \mathrm{g} / \mathrm{m}^{3}$ & $27 \mu \mathrm{g} / \mathrm{m}^{3}$ & $40 \mu \mathrm{g} / \mathrm{m}^{3}$ & $100 \mu \mathrm{g} / \mathrm{m}^{3}$ \\
2018 & January & $82 \mu \mathrm{g} / \mathrm{m}^{3}$ & $24 \mu \mathrm{g} / \mathrm{m}^{3}$ & $40 \mu \mathrm{g} / \mathrm{m}^{3}$ & $100 \mu \mathrm{g} / \mathrm{m}^{3}$ \\
2018 & February & $75 \mu \mathrm{g} / \mathrm{m}^{3}$ & $29 \mu \mathrm{g} / \mathrm{m}^{3}$ & $40 \mu \mathrm{g} / \mathrm{m}^{3}$ & $100 \mu \mathrm{g} / \mathrm{m}^{3}$ \\
2018 & March & $95 \mu \mathrm{g} / \mathrm{m}^{3}$ & $48 \mu \mathrm{g} / \mathrm{m}^{3}$ & $40 \mu \mathrm{g} / \mathrm{m}^{3}$ & $100 \mu \mathrm{g} / \mathrm{m}^{3}$ \\
2018 & April & $76 \mu \mathrm{g} / \mathrm{m}^{3}$ & $45 \mu \mathrm{g} / \mathrm{m}^{3}$ & $40 \mu \mathrm{g} / \mathrm{m}^{3}$ & $100 \mu \mathrm{g} / \mathrm{m}^{3}$ \\
\hline
\end{tabular}

Table 8. $\mathrm{SO}_{2}$ Found to be above permissible limits set by WHO in 2017 \& 2018. Below as per NAAQS.

\begin{tabular}{|c|c|c|c|c|c|}
\hline Year & Month & Shivajinagar & Pashan & WHO & NAAQS \\
\hline 2017 & May & $10 \mu \mathrm{g} / \mathrm{m}^{3}$ & $9 \mu \mathrm{g} / \mathrm{m}^{3}$ & $20 \mu \mathrm{g} / \mathrm{m}^{3}$ & $60 \mu \mathrm{g} / \mathrm{m}^{3}$ \\
\hline 2017 & June & $9 \mu \mathrm{g} / \mathrm{m}^{3}$ & $9 \mu \mathrm{g} / \mathrm{m}^{3}$ & $20 \mu \mathrm{g} / \mathrm{m}^{3}$ & $60 \mu \mathrm{g} / \mathrm{m}^{3}$ \\
\hline 2017 & July & $9 \mu \mathrm{g} / \mathrm{m}^{3}$ & $8 \mu \mathrm{g} / \mathrm{m}^{3}$ & $20 \mu \mathrm{g} / \mathrm{m}^{3}$ & $60 \mu \mathrm{g} / \mathrm{m}^{3}$ \\
\hline 2017 & August & $8 \mu \mathrm{g} / \mathrm{m}^{3}$ & $7 \mu \mathrm{g} / \mathrm{m}^{3}$ & $20 \mu \mathrm{g} / \mathrm{m}^{3}$ & $60 \mu \mathrm{g} / \mathrm{m}^{3}$ \\
\hline 2017 & September & $8 \mu \mathrm{g} / \mathrm{m}^{3}$ & $7 \mu \mathrm{g} / \mathrm{m}^{3}$ & $20 \mu \mathrm{g} / \mathrm{m}^{3}$ & $60 \mu \mathrm{g} / \mathrm{m}^{3}$ \\
\hline 2017 & October & $38 \mu \mathrm{g} / \mathrm{m}^{3}$ & $34 \mu \mathrm{g} / \mathrm{m}^{3}$ & $20 \mu \mathrm{g} / \mathrm{m}^{3}$ & $60 \mu \mathrm{g} / \mathrm{m}^{3}$ \\
\hline 2017 & November & $36 \mu \mathrm{g} / \mathrm{m}^{3}$ & $32 \mu \mathrm{g} / \mathrm{m}^{3}$ & $20 \mu \mathrm{g} / \mathrm{m}^{3}$ & $60 \mu \mathrm{g} / \mathrm{m}^{3}$ \\
\hline 2017 & December & $34 \mu \mathrm{g} / \mathrm{m}^{3}$ & $37 \mu \mathrm{g} / \mathrm{m}^{3}$ & $20 \mu \mathrm{g} / \mathrm{m}^{3}$ & $60 \mu \mathrm{g} / \mathrm{m}^{3}$ \\
\hline 2018 & January & $33 \mu \mathrm{g} / \mathrm{m}^{3}$ & $35 \mu \mathrm{g} / \mathrm{m}^{3}$ & $20 \mu \mathrm{g} / \mathrm{m}^{3}$ & $60 \mu \mathrm{g} / \mathrm{m}^{3}$ \\
\hline 2018 & February & $32 \mu \mathrm{g} / \mathrm{m}^{3}$ & $34 \mu \mathrm{g} / \mathrm{m}^{3}$ & $20 \mu \mathrm{g} / \mathrm{m}^{3}$ & $60 \mu \mathrm{g} / \mathrm{m}^{3}$ \\
\hline 2018 & March & $30 \mu \mathrm{g} / \mathrm{m}^{3}$ & $33 \mu \mathrm{g} / \mathrm{m}^{3}$ & $20 \mu \mathrm{g} / \mathrm{m}^{3}$ & $60 \mu \mathrm{g} / \mathrm{m}^{3}$ \\
\hline
\end{tabular}

Table 9 a. Shivajinagar area -Annual variation of Trees.

\begin{tabular}{ccccccc}
\hline Sr No. & Name of the Plant & $\mathrm{pH}$ & $\begin{array}{c}\mathrm{RWC} \text { value } \\
(\mathrm{mg} / \mathrm{gm})\end{array}$ & $\begin{array}{c}\text { Total Chlorophyll } \\
\left(\mathrm{mg} \mathrm{g}^{-1}\right)\end{array}$ & $\begin{array}{c}\text { Ascorbic acid content } \\
(\mathrm{mg} / \mathrm{gm})\end{array}$ & APTI \\
\hline 1 & $\begin{array}{c}\text { Polyalthia longifolia (Ashoka } \\
\text { tree) }\end{array}$ & 4.6 & 0.00809 & 0.1739 & 49.8 & 23.15 \\
2 & $\begin{array}{c}\text { Ficus racemosa (Umbar tree) } \\
4\end{array}$ & 4.8 & 0.00831 & 0.11928 & 66.4 & 31.93 \\
\hline
\end{tabular}

Table 9 b. Pashan area- Annual variation of Trees.

\begin{tabular}{ccccccc}
\hline Sr No. & Name of the Plant & $\mathrm{pH}$ & $\begin{array}{c}\mathrm{RWC} \text { value } \\
(\mathrm{mg} / \mathrm{gm})\end{array}$ & $\begin{array}{c}\text { Total Chlorophyll } \\
\left(\mathrm{mg} \mathrm{g}^{-1}\right)\end{array}$ & $\begin{array}{c}\text { Ascorbic acid content } \\
(\mathrm{mg} / \mathrm{gm})\end{array}$ & APTI \\
\hline $\begin{array}{c}\text { Polyalthia longifolia (Ashoka } \\
\text { tree) }\end{array}$ & 4.2875 & 0.304138 & 0.008625 & 99.6 & 42.81982 \\
2 & $\begin{array}{c}\text { Ficus racemosa }(\text { Umbar tree) } \\
2\end{array}$ & 4.2125 & 0.309288 & 0.008613 & 49.8 & 21.05207 \\
\hline
\end{tabular}

PM 10 concentration was highest with a value of 90 Ashoka tree with the concentration of PM 10 and PM 2.5. $\mu \mathrm{g} / \mathrm{m}^{3}$ at the polluted area (Shivaji Nagar) and the lowest 63 Polyalthia longifolia showed APTI 31.93 in the Shivajinagar $\mu \mathrm{g} / \mathrm{m}^{3}$ at the cleaner area (Pashan). This is attributed to high area and 42.81 in Pashan, indicating that the Ashoka tree is traffic density and movement in the Shivajinagar area, as tolerant to Air Pollution. The higher value of APTI may be shown in Fig. 3. The air pollution tolerant index denotes the due to the increased production of ascorbic acid. ${ }^{[23]}$

potential of a plant to combat air pollution. ${ }^{[27]}$ Fig. 3 Ficus racemosa showed an APTI value of 23.15 in the represents APTI values and the average concentration of PM Shivajinagar area and 21.05 in Pashan, showing it to be 10 and PM 2.5. Fig. 3(a) compares the APTI value of the intermediate to air pollution, as shown in Fig. 3(b). The study 


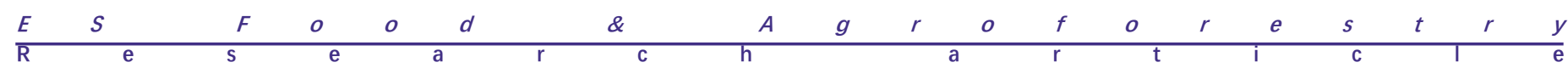

revealed that Ficus racemosa was intermediate to air pollution in Shivajinagar and Pashan, and Polyalthia longifolia was tolerant in both areas. Higher APTI values in the polluted environment as compared to the controlled environment. This could be due to different environmental conditions related to a load of air pollutants and climate. ${ }^{[28]}$ Moreover, the sensitivity of a plant species varies from area to area as tolerance of plants towards air pollutants is specific to a site and depends on the type and level of pollution and other atmospheric conditions. ${ }^{[29]}$ There is a reduction in $\mathrm{pH}$, R.W.C., Ascorbic acid content, and Total chlorophyll content (Tables 9a \& b) was observed more in Shivajinagar (polluted) area than in Pashan (comparatively cleaner area).

Herein we compare the observed experimental values with the standard. From careful observation and analysis with standard parameters, it is observed that air quality in the Shivajinagar area in all seasons deviates to greater extents from the standard norms. Therefore, it is concluded that the air quality of the suburban area of Shivajinagar is highly polluted and unhygienic. On the other hand, when we compare the air quality parameters of the suburban area of Pashan with standard norms, we observe that the values are very much close to the standard norms as specified by WHO and NAAQS standards (Fig. 4).

\section{Conclusion}

Particulate matter (PM $10 \& 2.5$ ) was found to be above the permissible limit in the winter and summer season. In contrast, $\mathrm{SO}_{\mathrm{x}}, \mathrm{NO}_{2}$ was found to be within permissible limits as per standards prescribed by WHO \& NAAQS. Shivajinagar area was found to be more polluted than the Pashan area. APTI (Air quality tolerance index) of plants, four parameters, namely ascorbic acid, Total Chlorophyll, Relative water content, $\mathrm{pH}$ was computed in the formula to get susceptibility levels of plants. A considerable reduction has been observed in total Chlorophyll, ascorbic acid content, leaf $\mathrm{pH}$, relative water content, APTI of plant samples. However, the magnitude of reduction of these parameters was higher in polluted areas. Results also indicate that the air pollution tolerance index (APTI) is higher in polluted locations than in cleaner environments. Hence, it is stressed that Polyalthia longifolia, along with Ficus racemosa, can serve as a barrier and sink to control air pollution and can be used as one of the mitigating measures to curb air pollution roadsides in traffic areas. Thus it is concluded that the air quality of the Pashan suburban area is suitable for human life and hygiene. This study reflects that the air quality of Pune city in two different areas varies to a large extent.

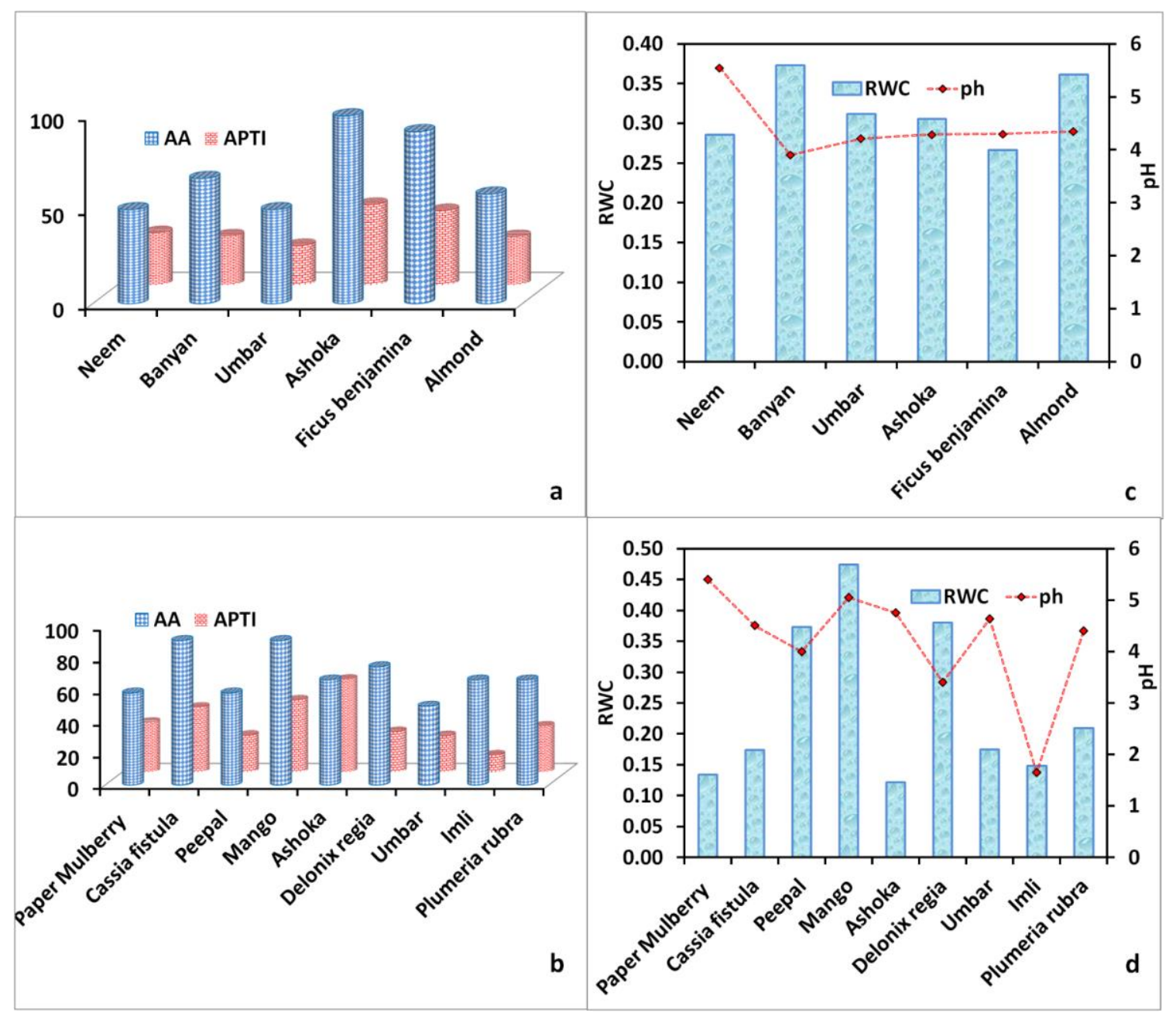

Fig. 4 Variation of biochemical parameters and APTI in different plants at Shivaji nagar (a) \& (c) and Pashan (b) \& (d). 


\section{Acknowledgements}

The authors are grateful to Director, IITM, Pune and Principal, Abeda Inamdar College for their support.

\section{Conflict of Interest}

There is no conflict of interest.

\section{Supporting Information}

Not Applicable.

\section{References}

[1] S. Amina, Case study for Bhopal Gas Tragedy, 2020, https://www.researchgate.net/publication/343600799_Case_stud y_for_Bhopal_Gas_Tragedy.

[2] I. Manisalidis, E. Stavropoulou, A. Stavropoulos, E. Bezirtzoglou, Front Public Health, 2020, 8, doi: 10.3389/fpubh.2020.00014.

[3] M. V. Nikolic, V. Milovanovic, Z. Z. Vasiljevic, Z. Stamenkovic, Sensors (Basel), 2020, 20, 6694, doi:10.3390/s20226694.

[4] R. Rai, M. Rajput, M. Agrawal, S. Agrawal, J. Sci. Res., 2011, 55, 77-102.

[5] G. Beig, S.K. Sahu, V. Singh, S. Tikle, B.S. Sobhana, K. Ramakrishna, G. Prashant, A. Rathod, B.S. Murthy., Sci. Total Environ. $\quad 2020, \quad 709, \quad 136126, \quad$ doi: 10.1016/j.scitotenv.2019.136126.

[6] T. Gordon, K. Balakrishnan, S. Dey, Environ. Int., 2018, 119 , 100-108, doi: 10.1016/j.envint.2018.06.013

[7] I. Manisalidis, E. Stavropoulou, A. Stavropoulos, E. Bezirtzoglou, Front Public Health, 2020, 8, 14, doi: 10.3389/fpubh.2020.00014.

[8] M. Nimisha, S. Joseph, Int. j. botany stud., 2019, 4, 110-114. [9] W.H. Smith, J. Air Pollut. Control Assoc., 1976, 26, 753-766, doi: 10.1080/00022470.1976.10470310.

[10] H. Singh, M. Yadav, N. Kumar, A. Kumar, M. Kumar, PLoS One, 2020, 15, 1-20, doi: 10.1371/journal.pone.0227380.

[11] L. Girish, K. Krishnakutty, S. Vaidya, Int. J. Curr. Res., 2017, 9, 57807-57811.

[12] V. Pathak, B. D. Tripathi, V. K. Mishra, Urban For. Urban Green., 2011, 10, 61-66, doi: 10.1016/j.ufug.2010.06.008.

[13] G. Mahecha, B.R. Bamniya, N. Nair, D. Saini, Int. J. Innov. Res. Sci. Eng. Technol., 2013, 2, 7927-7929.

[14] P. S. Lakshmi, K. L. Sravanti, N. Srinivas, The Ecoscan, 2008, 2, 203-206.

[15] A. K. Tripathi, M. Gautam, J. Environ. Biol., 2007, 28, 127-132.

[16] K. Kushreshtha, A. Rai, C.S. Mohanty, R.K. Roy, S.C. Sharma, Int. J. Environ. Res., 2009, 3, 137-142, doi: 10.22059/ijer.2009.42

[17] P.D. Safai, S. Kewat, P.S. Praveen, P.S.P. Rao, G.A. Momin, K. Ali, P.C.S. Devara, Atmos. Environ., 2007 41, 2699-2709, doi: 10.1016/j.atmosenv.2006.11.044

[18] V. Anand, N. Korhale, A. Rathod, G. Beig, Environ. Pollut., 2019, 254, 113026, doi: 10.1016/j.envpol.2019.113026.

[19] S.K. Peshin, P. Sinha, A. Bisht, Mausam, 2017, 68, 111-
118, doi: https://doi.org/10.5572/ajae.2017.11.4.322

[20] G. Beig, D.M. Chate, S.D. Ghude, A.S. Mahajan, R. Srinivas, K. Ali, S.K. Sahu, N. Parkhi, D. Surendran, H.R. Trimbake, Atmos. Environ., 2013, 80, 455-463, doi: doi: 10.1016/j.atmosenv.2013.08.012.

[21] B.S. Murthy, R. Latha, A. Tiwari, A. Rathod, S. Singh, G. Beig, J. Atmos. Solar-Terrestrial Phys., 2019, 197, 105157, doi: 10.1016/j.jastp.2019.105157.

[22] S. K. Singh, D.N. Rao, M. Agrawal, J. Pandey, D. Naryan, J. Environ. Manage., 1991, 32, 45-55, doi: 10.1016/S0301-4797(05)80080-5.

[23] K. Walia, R. K. Aggrawal, S. K. Bhardwaj, Int. J. Curr. Microbiol. Appl. Sci., 2019, 8, 2498-2508, doi: 10.20546/ijcmas.2019.803.296.

[24] Deepika, P. Gour, A. K. Haritash, Int. J. Environ. Sci., 2016, 6, 979-986, doi: 10.6088/ijes.6092.

[25] S. Acharya, R. C. Jena., S. J. Das, C. Pradhan, P. K. Chand,

J. Environ. Biol., 2017, 38, 1397-1403, doi: $10.22438 / \mathrm{jeb} / 38 / 6 / \mathrm{MS}-183$.

[26] I. Manisalidis, E. Stavropoulou, A. Stavropoulos, E. Bezirtzoglou, Front public health., 2020, 8, 14, doi: doi: 10.3389/fpubh.2020.00014

[27] A. Begum, S. Harikrishna, Indian J. Chem., 2010, 7, 151157, doi: 10.1155/2010/398382

[28] P. Agbaire, E. Esiefarienrhe, J. Appl. Sci. Environ. Manag., 2009, 13, 11-14, doi: 10.4314/jasem.v13i1.55251.

[29] M.J. Noor, S. Sultana, S. Fatima, M. Ahmad, M. Zafar, M. Sarfraz, M.A. Balkhyour, S. Z. Safi, M. A. Ashraf, Env. Geochem Heal., 2015, 37, 441-55, doi: 10.20546/ijcmas.2018.710.010.

[30] D. Aron, Plant Physiology., 1949, 24, 1-15, doi: 10.1104\%2Fpp.24.1.1.

[31] A. Singh, Practical plant physiology, Amar Singh, New Delhi, Kalyani, 1977.

[32] B. Åberg Ascorbic acid. In: Åberg B. et al. (eds) Aufbau • Speicherung - Mobilisierung und Umbildung der Kohlenhydrate/Formation • Storage • Mobilization and Transformation of Carbohydrates. Handbuch der Pflanzenphysiologie/Encyclopedia of Plant Physiology, vol 6. Springer, Berlin, Heidelberg, 1958, doi: 10.1007/978-3-642-94731-5 19.

\section{Author Information}

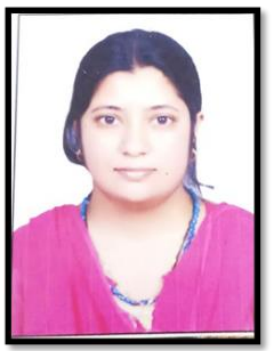

Erum $\boldsymbol{F} \boldsymbol{H}$ Kazi, is an Assistant Professor, in the Department of Environmental Science Abeda Inamdar Senior College from last 08 years. She has completed MSc in Environmental Science and qualified UGC NET. She had attended various Orientation, Refresher Courses, Conferences, FDP, seminars, Workshops and has published many papers, posters in Conferences. She has successfully completed diploma in Green teacher's from CEE (Center for 


$r$

r

${ }^{o}$

$r$

Green Chemistry Approach. He has also taken part in various National Conferences and Symposium and presented his research work and also delivered lectures in various lecture competitions and have been a winner.

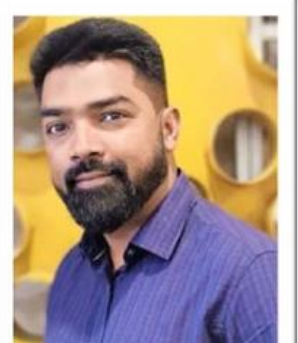

Dr. Yaseen Ismail Shaikh is an Assistant Professor of Organic Chemistry in the Department of Chemistry at Abeda Inamdar Senior College, Camp Pune 411001. He obtained his Ph.D. in 2018 from S.P. Pune University, Pune. He had also qualified National Eligibility Test (NET) with 28th rank in L.S. in "Council of Scientific \& Industrial Research Human Resource-University Grant Commission test for J.R.F. His research focuses on Synthetic Organic Chemistry, Nanotechnology, Computational Chemistry, Medicinal Chemistry, Transition Metal Catalysis, Multicomponent Reactions, and Green Chemistry. He is also a Recognized Research Guide for M.Phil in Chemistry. Affiliated to S.P. Pune University Pune. He has more than 25 scientific publications in International Journals. He has also presented Research papers in various International and National conferences. He is a member of several Chemical Societies. He is also a recipient of the Best Teacher Award.

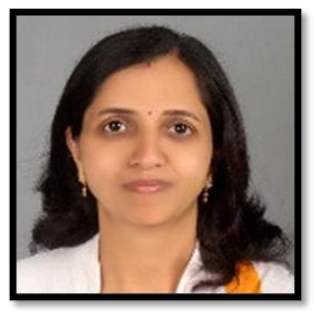

Dr. Suvarna Tikle, is a Professional Environmentalist with 12 years of experience in Air Quality, Water quality, ARC GIS, RS and modeling. Worked as Professor in Savitri bai Phule University in Department of Environmental Science.

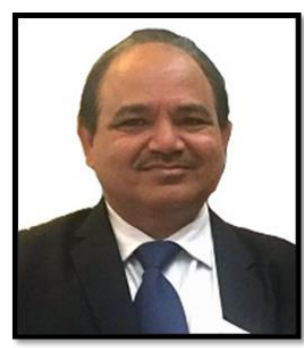

Dr Gufran Beig, is the Joint Director at Indian Institute of Tropical Meteorology. He is an eminent scientist and his research is focused in Atmospheric Chemical-Transport Modeling, Assessment of Temperature Trend in the Middle Atmosphere, Atmospheric Chemistry and Changing Climate and Impact on Health, Development of the System of Air Quality Forecasting and Research for Urban Cities.

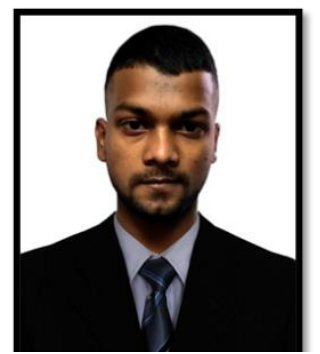

Mr. Viquar Sameer Shaikh is a Research Scholar in the Department of Chemistry, Nowrosjee Wadia College Pune-1. He has obtained his B.Sc. (Chemistry) and M.Sc. (Physical Chemistry) from S.P. Pune University Pune. $\quad \mathrm{He}$ is researching Pharmaceutical co-crystals at NCL, Pune. He is also researching on the potential inhibitors for COVID-19 using Computer-Aided Drug Design. Also organic synthesis i.e. on Multicomponent reaction towards a

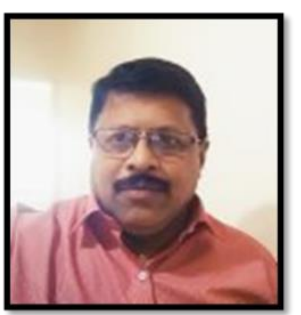

Dr Satish D Kulkarni, is the Director of Environmental Science Department, New Arts, Commerce and Science College Ahmednagar. He has 17 years of experience in field of Environmental Science, PhD in Environmental Biotechnology. Presently he is working as an Associate professor in New Arts, Commerce and Science College Ahmednagar.

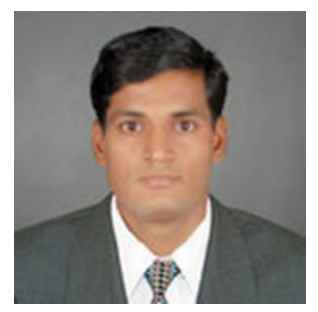

Dr. Vinayak Popat Dhulap, Ph.D (Dr. BAMU, Aurangabad), M.Sc (SU Kolhapur), UGC-NET, UGC - SET in Environmental Science. He is working as Assistant Professor and Head, Department of Environmental Science, School of Earth Sciences, Punyashlok Ahilyadevi Holkar Solapur University Solapur (MS) India. He has 13 years of teaching and research experience; He is reviewer for many research journals like Ecological Processes, Measurement, Ecological Engineering, Energies, Journal of Geosciences research, Sustainable materials and technologies etc. He is coordinator for Centre of BARTI and TRTI Competitive Examination also coordinator for Skill Course - Environmental Informatics and Co-Cordinator for Agrotourism, PAH Solapur University Solapur. Dhulap has forty two (42) peer reviewed articles and has authored 3 books 3 chapters and he has presented research papers more than 30 National and International conferences. He has organized few research conferences, workshops on Environment and allied subjects at Solapur University. Dhulap has mobilized funds through many Environmental Consultancy Project Work and research projects. He has completed 4 major / minor research projects. He has Lifetime Membership of the Indian Science Congress and World Wide Funds. He is member for various committees EIA Expert District Level Committee, Public Biodiversity Committee Solapur, Environmental Expert in Heritage Conservation Committee etc.

Publisher's Note: Engineered Science Publisher remains neutral with regard to jurisdictional claims in published maps and institutional affiliations. 